\title{
Protein phosphatase 2A Ca regulates proliferation, migration, and metastasis of osteosarcoma cells
}

\author{
Di Yang ${ }^{1,2}$, Hirohiko Okamura², Hiroyuki Morimoto ${ }^{3}$, Jumpei Teramachi ${ }^{2}$ and Tatsuji Haneji
}

Osteosarcoma is the most frequent primary bone tumor. Serine/threonine protein phosphatase 2A (PP2A) participates in regulating many important physiological processes, such as cell cycle, growth, apoptosis, and signal transduction. In this study, we examined the expression and function of PP2A Ca in osteosarcoma cells. PP2A Ca expression was expected to be higher in malignant osteosarcoma tissues. PP2A Ca expression level and PP2A activity was higher in malignant osteosarcoma LM8 cells compared with that in primary osteoblasts and in the osteoblast-like cell line MC3T3-E1. Okadaic acid, an inhibitor of PP2A, reduced cell viability and induced apoptosis in LM8 cells. PP2A Ca-knockdown LM8 cells (shPP2A) exhibited less striking filopodial and lamellipodial structures than that in original LM8 cells. Focal adhesion kinase phosphorylation and NF- $K B$ activity decreased in shPP2A-treated cells. Sensitivity to serum deprivation-induced apoptosis increased in shPP2A-treated cells, accompanied by a lower expression level of anti-apoptotic BCL-2 in these cells.

Reduction of PP2A Ca resulted in a decrease in the migration ability of LM8 cells in vitro. Reduction in PP2A Ca levels in vivo suppressed proliferation and metastasis in LM8 cells. PP2A Ca expression was also higher in human osteosarcoma MG63 and SaOS-2 cells than that in primary osteoblasts and MC3T3-E1 cells, and reduction in PP2A Ca levels suppressed the cell proliferation rate and migration ability of MG63 cells. These results indicate that PP2A Ca has a critical role in the proliferation and metastasis of osteosarcoma cells; therefore, its inhibition could potentially suppress the malignancy of osteosarcoma cells.

Laboratory Investigation (2016) 96, 1050-1062; doi:10.1038/labinvest.2016.82; published online 29 August 2016

Osteosarcoma is the most frequent primary malignant tumor of the skeleton, usually developing before 30 years of age. ${ }^{1,2}$ Osteosarcoma grows aggressively at the primary site and often develops subclinical micrometastasis in the lung before diagnosis, with consequently poor prognosis. The usual treatment for osteosarcoma is the complete removal of the primary lesion by wide surgical resection and aggressive adjuvant chemotherapy. ${ }^{3,4}$ Despite progress in chemotherapy for osteosarcoma, distant metastasis is the major cause of mortality. Therefore, the prevention of pulmonary metastases during the early stage of tumor development is critical to improve the prognosis for patients with osteosarcoma.

The LM8 osteosarcoma cell line was established from murine Dunn osteosarcoma cells inoculated i.v. into male $\mathrm{C} 3 \mathrm{H}$ mice through the tail vein. ${ }^{5,6}$ Compared with Dunn cells, LM8 cells exhibit more striking filopodial and lamellipodial structures, and have higher proliferation rates and metastatic potentiality to the lung. ${ }^{6}$ LM8 cells were also reported to possess activated focal adhesion kinase (FAK) and high NF- $\kappa \mathrm{B}$ activity. ${ }^{7,8}$ Therefore, LM8 cells could be a useful tool to study the mechanisms involved in proliferation and metastasis of osteosarcoma.

Protein phosphatases regulate many key cellular processes including glycogen metabolism, cell cycle, and protein synthesis. Based on their biochemical parameters, serine/ threonine phosphatases were initially divided into two classes: type 1 phosphatases (PP1) and type 2 phosphatases (PP2). ${ }^{9,10}$ Furthermore, PP2 comprises three enzymes (PP2A, PP2B, and $\mathrm{PP} 2 \mathrm{C}$ ) that can be distinguished by their cation requirements. ${ }^{9,10}$ Of these, PP2A refers to a large family of heterotrimeric phosphatases in eukaryotic cells. ${ }^{11} \mathrm{PP} 2 \mathrm{~A}$ comprises a structural A subunit, a regulatory $\mathrm{B}$ subunit, and a catalytic $\mathrm{C}$ subunit. ${ }^{12,13}$ The catalytic $\mathrm{C}$ subunit is divided into two isoforms, $\mathrm{C} \alpha$ and $\mathrm{C} \beta$. $\mathrm{C} \alpha$ transcripts are about 10-fold

\footnotetext{
${ }^{1}$ Department of Endodontics, School of Stomatology, China Medical University, Shenyang, China; ${ }^{2}$ Department of Histology and Oral Histology, Institute of Biomedical Sciences, Tokushima University Graduate School, Tokushima, Japan and ${ }^{3}$ Department of Anatomy, School of Medicine, University of Occupational and Environmental Health, Kitakyushu, Fukuoka, Japan

Correspondence: Dr D Yang, DDS, PhD or Professor H Okamura, DDS, PhD, Department of Histology and Oral Histology, Institute of Biomedical Sciences, 3-18-15, Kuramoto, Tokushima 770-8504, Japan.
}

E-mail: missyangdi@hotmail.com or okamura@tokushima-u.ac.jp

Received 29 January 2016; revised 20 June 2016; accepted 23 June 2016 
more abundant in many tissues. ${ }^{14}$ A combinatorial assembly of $\mathrm{A}, \mathrm{B}$, and $\mathrm{C}$ subunits permits the formation of many distinct PP2A complexes, which have been implicated in the control of various cellular processes, including cell proliferation, survival, adhesion, and cytoskeletal dynamics. ${ }^{12}$ Knockout mice lacking the PP2A C subunit $\alpha$-isoform (PP2A C $\alpha$ ) showed embryonic lethality and an absence of mesoderm induction, ${ }^{14,15}$ indicating that $\mathrm{PP} 2 \mathrm{~A} \mathrm{C} \alpha$ has a pivotal role in the development of mesenchymal cells including osteoblasts. We have previously reported that okadaic acid (OA), a potent inhibitor of PP2A, induces apoptosis in the human osteosarcoma cell lines, MG63 and SaOS-2, ${ }^{16-18}$ suggesting that PP2A is involved in proliferation and apoptosis of osteosarcoma cells. Furthermore, we also demonstrated that PP2A C $\alpha$ has important roles in bone formation and osteoblast differentiation through bone-specific genes including Osterix. ${ }^{19,20}$ PP2A C $\alpha$ is also involved in the osteoblast function of controlling osteoclastogenesis. ${ }^{21} \mathrm{PP} 2 \mathrm{~A} \mathrm{C} \alpha$ is a key regulator of osteoblast differentiation and function, which suggests that it also has an important role in the proliferation and metastasis of osteosarcoma cells, because osteosarcoma cells are derived from osteoblasts with malignant mutations. However, the role of PP2A C $\alpha$ in osteosarcoma cells has not been investigated.

Diverse conditions such as serum deprivation induce apoptosis through the intrinsic mitochondrial pathway. BCL-2 family proteins comprise anti-apoptotic factors (BCL-2, BCL-XL) and pro-apoptotic factors (BAX, BIM). These members are critical regulators of cell survival and death, controlling the integrity of the mitochondrial membrane. ${ }^{22}$ The expression patterns of BCL-2 family proteins elicit the release of cytochrome $\mathrm{C}$ from mitochondria, which in turn induces caspase- 3 activation and poly ADP-ribose polymerase (PARP) cleavage. ${ }^{22-24}$ Therefore, the ratio between the antiapoptotic and pro-apoptotic BCL-2 family proteins determines the susceptibility of cells to death signals.

In this study, we examined the role of PP2A C $\alpha$ in the proliferation, migration, and metastasis of osteosarcoma cells in vitro and in vivo.

\section{MATERIALS AND METHODS Materials}

PP2A C $\alpha$, caspase-3, PARP, Bcl-2, Bim, Bax, and Bcl-xL antibodies were purchased from Cell Signaling Technology (Danvers, MA, USA). $\beta$-actin and phospho-FAK antibodies were purchased from Sigma-Aldrich (St Louis, MO, USA). Proliferating cell nuclear antigen (PCNA) antibody was purchased from DAKO (Glostrup, Denmark). Rhodaminephalloidin was purchased from Life Technologies (Grand Island, NY, USA). OA was purchased from Wako (Osaka, Japan). The other materials used were of the highest grade available commercially.

\section{Immunohistochemical Analysis}

Osteosarcoma sections on a tumor tissue slide array (Super Bio Chips, Seoul, Korea) were analyzed by immunohisto- chemistry using PP2A C $\alpha$ antibody. After washing in phosphate-buffered saline (PBS), the sections were incubated with anti-rabbit biotinylated secondary antibody and avidinbiotin-peroxidase complex (Nakalai Tesque, Kyoto, Japan). The reaction was then developed with a peroxidase stain $\mathrm{DAB}$ kit (Nakalai Tesque). Specificity of immune reactivity of PP2A $\mathrm{C} \alpha$ antibody was confirmed using an ileum section stained with this antibody as positive and negative controls. As a negative control, primary PP2A $\mathrm{C} \alpha$ antibody was replaced with non-immune normal rabbit serum. Histopathological information for the tissues on the slide is provided online (http://www.novusbio.com/Osteosarcoma-Tissue-Micro-Array_ NBP2-30289.html).

\section{Cell Culture}

Mouse preosteoblastic MC3T3-E1 and osteosarcoma LM8 cells were obtained from Riken Cell Bank (Tsukuba, Japan). Human osteosarcoma cell lines MG63 and SaOS-2 were purchased from the American Type Culture Collection (Rockville, MD, USA). Primary calvarial osteoblasts were isolated from 3-day-old mice. Cells were cultured in $\alpha$-MEM supplemented with $10 \%$ fetal bovine serum (FBS) at $37^{\circ} \mathrm{C}$ under a humidified atmosphere of $5 \% \mathrm{CO}_{2}$.

\section{SDS-PAGE and Western Blot Analysis}

Protein samples and pre-stained molecular weight markers (Bio-Rad, Hercules, CA, USA) were separated by SDS-PAGE and transferred to PVDF membranes (Millipore, Medford, MA, USA). The membranes were incubated overnight at $4{ }^{\circ} \mathrm{C}$ in blocking solution containing specific antibodies (diluted at 1:1000). After being washed in PBS-Tween, the membranes were incubated with horseradish peroxidase-conjugated secondary antibodies (diluted at 1:5000). The proteins recognized by the antibodies were visualized with an Immobilon western detection kit (Millipore).

\section{Serine/Threonine Protein Phosphatase Activity Assays}

The activity of serine/threonine phosphatase was determined using a nonradioactive molybdate dye-based phosphatase assay kit (Promega, Madison, WI, USA) according to the manufacturer's recommendations. Absorbance at $630 \mathrm{~nm}$ was measured using an iMark Absorbance Reader (Bio-Rad, Hercules, CA, USA) and the amount of phosphate released was calculated using the standard curve.

\section{Cell Viability Assay}

The cells were seeded into a 96-well plate at a density of $0.2 \times 10^{4}$ cells/well. After attachment, the cells were treated with OA for $24 \mathrm{~h}$. Cell viability was measured using a Cell Counting Kit (Dojindo, Kumamoto, Japan).

\section{Transfection}

A pGL4 expression vector (Promega) was transfected into the cells using the Lipofectamine reagent (Life technologies). The cells were cultured with $600 \mu \mathrm{g} / \mathrm{ml}$ Geneticin (G418) for 
10 days. Positive clones stably expressing the luciferase gene were selected and infected with MISSION shRNA lentiviral transduction particles for PP2A C $\alpha$ (NM_019411, SigmaAldrich) according to the manufacturer's directions, and the stable clones (shPP2A) were obtained after puromycin $(5 \mu \mathrm{g} / \mathrm{ml})$ selection. MISSION Non-Target shRNA lentiviral transduction particles were used as negative control (shCont). To detect bioluminescence in cultured cells using an IVIS spectrum (Caliper, PerkinElmer), D-luciferin $(10 \mu \mathrm{g} / \mathrm{ml})$ was added to the culture dish. The luciferase produced by viable LM8 cells reacts with luciferin and generates bioluminescence. Bioluminescence was calculated by integrating the bioluminescence signal between 5 and 10 min after i.v. injection or after direct addition to the cultured cells. For transient silencing of PP2A C $\alpha$, MG63 cells were transfected with validated stealth siRNA targeting human PP2A C $\alpha$ (\#HSS108358, Invitrogen) with Lipofectamine 2000 (Invitrogen) (siPP2A). Nonspecific siRNA (Invitrogen) was also transfected as a negative control (siCont).

\section{Immunocytochemistry}

Cells were grown on sterile $18-\mathrm{mm}$ round glass coverslips placed in $60-\mathrm{mm}$ plastic dishes. After blocking, the coverslips were incubated for $30 \mathrm{~min}$ with a rhodamine-phalloidin or an anti-phospho-FAK antibody diluted at 1:200 in 4\% BSA. After three washes with PBS, they were incubated with Alexa Fluor 488 goat anti-mouse IgG (Invitrogen). After incubation with $10 \mu \mathrm{g} / \mathrm{ml}$ Hoechst 33342 for $30 \mathrm{~min}$, the coverslips were mounted with fluorescent mounting medium (Dako, Glostrup, Denmark). The cells were examined under an Olympus BX50 microscope equipped with epifluorescence illumination and a U-MNIBA filter for green, blue, and red fluorescence (Olympus, Tokyo, Japan). Photomicrographs were recorded on a computer (Olympus, DP70-WPCXP).

\section{Luciferase Assay}

An NF- $\kappa$ B luciferase reporter was obtained from Stratagene (La Jolla, CA, USA). Cells were transfected with NF- $\kappa \mathrm{B}$ reporter vector with the aid of Lipofectamine LTX reagent (Life technologies). The cells were cultured for another $24 \mathrm{~h}$ and total cell lysates were prepared using the Dual-Glo Luciferase Assay System (Promega) and assessed for luciferase activity. The efficiency of transfection was standardized by co-transfection with pTK-Renilla (Promega).

\section{In Vitro Migration Assay}

Migration was analyzed in a Boyden chamber assay using Falcon cell culture inserts (pore size $8 \mu \mathrm{m}$; Becton Dickinson, Frankinson Lakes, NJ, USA). The upper chamber of the insert was filled with $500 \mu \mathrm{l}$ serum-free medium containing cells and $1 \mathrm{ml}$ serum-containing medium was added into the lower chamber. After incubation for $24 \mathrm{~h}$, the remaining cells on the upper surface of the membrane were removed and the migrated cells on the lower surface were fixed and stained with trypan blue. Cells that had penetrated through the
$8 \mu \mathrm{m}$-pore culture inserts were counted using a light scope. For the scratch assay, cells were grown to confluence on culture dishes and a scratch was made in the cell monolayer using a sterile pipette tip. Cells were washed with PBS and then cultured for another $12 \mathrm{~h}$. Cells were photographed before and after treatment and gap widths were measured using ImageJ software.

\section{Seahorse XF24 Metabolic Flux Analysis}

To measure the mitochondrial bioenergetics profile in live cells, a Seahorse Bioscience XF24 Extracellular Flux Analyzer was used (Seahorse Bioscience, North Billerica, MA, USA). Cells were plated on XF24 plates and allowed to settle for $24 \mathrm{~h}$. The cells were transferred into the recommended $\mathrm{XF}$ Assay medium (phenol red-free DMEM assay medium containing $2 \mathrm{mM}$ Glutamax, $1 \mathrm{mM}$ sodium pyruvate, $25 \mathrm{mM}$ glucose and $1.85 \mathrm{~g} / 1 \mathrm{NaCl}, \mathrm{pH} 7.4$ ) and maintained at $37^{\circ} \mathrm{C}$ in a non- $\mathrm{CO}_{2}$ incubator for $1 \mathrm{~h}$ before performing the assay. Dual-analyte sensor cartridges were preincubated overnight in XF24 Calibrant solution (Seahorse Bioscience). Mitochondrial function was interrogated by the sequential injection of oligomycin A $(1 \mu \mathrm{M})$, FCCP $(0.5 \mu \mathrm{M})$ and rotenone $(2 \mu \mathrm{M})$ in combination with antimycin A $(1 \mu \mathrm{M})$. Oxygen consumption rate (OCR, pmol $\mathrm{O}_{2} / \mathrm{min}$ ) was automatically calculated and recorded by the Seahorse XF24 software.

\section{Measurement of Phosphatidylserine (PS) Exposure}

The level of PS exposure was determined by measuring the extent of annexin V-fluorescein isothiocyanate (FITC) binding using an apoptosis detection kit (Beckman Coulter, Miami, FL, USA). Briefly, the cells were collected, washed with PBS, and resuspended in $1 \times$ binding buffer at a concentration of $1 \times 10^{6}$ cells $/ \mathrm{ml}$. Overall, $5 \mu \mathrm{l}$ of annexin V-FITC and $2.5 \mu \mathrm{l}$ of propidium iodide (PI, final conc. $250 \mu \mathrm{g} / \mathrm{ml}$ ) were added to $100 \mu \mathrm{l}$ of the cell suspension. After incubation for $15 \mathrm{~min}$ on ice, the cells were sequentially analyzed by cell analyzer (EPICS XL-MCL, Beckman Coulter).

\section{Subcutaneous and Intratibial Transplantation Studies}

Six- to eight-week-old BALB/c mice were purchased from Japan SLC (Hamamatsu, Japan). All mice studied were reared in our specific pathogen-free mouse colony and given food and water ad libitum. Experiments were humanely conducted under the regulation and permission of the Animal Care and Use Committee of the University of Tokushima, Tokushima, Japan (toku-dobutsu 12031). For the proliferation studies, $1 \times 10^{6}$ cells were mixed with an equal volume of BD Matrigel basement membrane and subcutaneously transplanted into the back of five mice in each group. For the metastasis studies, $1 \times 10^{6}$ cells were injected intraosseously into the proximal part of the tibia shaft of five mice in each group. These subcutaneous and intratibial transplantations were independently performed three times. Cell growth and metastasis were monitored every 2 days using IVIS spectrum (Caliper, PerkinElmer). The mice were anesthetized and injected IP 
with luciferin at $150 \mathrm{mg} / \mathrm{kg}$ in a volume of $150 \mu \mathrm{l}$. Images were recorded and then analyzed using Living Image software (Xenogen Corporation, Alameda, CA, USA).

\section{Histopathology}

Lung and liver tissues were collected from the mice and fixed with $4 \%$ paraformaldehyde overnight at $4{ }^{\circ} \mathrm{C}$. Samples were washed with PBS three times for $1 \mathrm{~h}$ at room temperature. After dehydration, samples were embedded in paraffin blocks. Sections were stained with hematoxylin and eosin following standard procedures and were analyzed by immunohistochemistry using PCNA antibody.

\section{Statistical Analysis}

All data were expressed as means \pm s.d. and a minimum of three independent experiments were performed for each assay. Analysis of variance (ANOVA) was used for statistical analysis. Statistical significance was indicated with ' ${ }^{\text {(*) }}(P$ value $<0.05)$ or $^{(* *)}(P$ value $<0.01)$.

\section{RESULTS}

\section{Osteosarcoma Shows Various Expression Patterns of PP2A Ca}

PP2A C $\alpha$ expression was examined in osteosarcoma tissue samples. PP2A $\mathrm{C} \alpha$ was detected distributed throughout the cell cytoplasm, and/or nucleus and high-PP2A C $\alpha$ expression levels were observed in 53\% (26/49) of the analyzed osteosarcoma tissues (Figure 1a, D-F) compared with nonmalignant granulation tissue (Figure 1a, A). Some osteosarcoma tissue samples had lower $(33 \%, 16 / 49)$ or similar levels $(14 \%, 7 / 49)$ of PP2A C $\alpha$ expression (Figure 1a, B, and C, respectively) compared with non-malignant granulation tissue. Specificity of immune reactivity of the antibody was examined via staining of the ileum section with (positive) or without (negative) the primary antibody. We also examined PP2A $\mathrm{C} \alpha$ expression in primary osteoblasts, osteoblastic MC3T3-E1, and malignant osteosarcoma LM8 cells. The expression of PP2A C $\alpha$ was significantly higher in LM8 cells compared with that in other cells (Figure 1b). In agreement with the result of western blot analysis of PP2A C $\alpha$ expression, PP2A activity was higher in LM8 cells (Figure 1c).

\section{OA Induces Apoptosis in LM8 Cells}

To examine whether PP2A activity is involved in cell viability, LM8 cells were treated with OA, a potent inhibitor of PP2A. Figure 2a shows the phase-contrast photomicrographs of the LM8 cells treated with OA for $24 \mathrm{~h}$. Cell rounding, shrinking, and floating were caused in a dose-dependent manner by OA treatment. Treatment with OA decreased cell viability in LM8 cells (Figure 2b). We further examined the effect of OA on cell viability using the stably transfected luciferase-expressing LM8 cells (LM8-Luc). As shown in Figure 2c (left panel), treatment with luciferin (Luci), a substrate of luciferase, allows us to observe living cells, with corresponding bioluminescence intensity in LM8-Luc cells, but not in original LM8 cells. We treated
LM8-Luc cells with various concentrations of OA for $24 \mathrm{~h}$. Figure $2 \mathrm{c}$ (right panel) shows that OA decreased bioluminescence intensity in LM8-Luc cells in a dose-dependent manner, which is in agreement with the results of the cell morphological change and viability assay. To examine whether $\mathrm{OA}$ induces apoptosis in LM8 cells, western blot analysis using caspase-3 antibody was performed. Cleaved forms of caspase- 3 were detected in the cells treated with 20 and $50 \mathrm{nM} \mathrm{OA}$ (Figure 2d).

\section{Silencing of PP2A Ca Changes Cell Morphology and Suppresses Cell Growth in LM8 Cells}

PP2A C $\alpha$-knockdown cells were established by transfecting shRNA into luciferase-expressing LM8 cells. Figure 3a shows that shPP2A cells express lower levels of PP2A C $\alpha$ compared with that in shCont cells. In agreement with this result, PP2A activity was lower in shPP2A cells than in shCont cells (Figure $3 \mathrm{~b}$ ). As shown in Figure $3 \mathrm{c}$, shCont cells and the original LM8 cells showed considerably higher numbers of filopodia per cell. However, shPP2A cells had a comparatively spindle-shaped morphology with a smooth cell surface. One thousand cells were plated in $35 \mathrm{~mm}$ plastic dishes, and cell numbers were measured every day for 3 days (Figure $3 \mathrm{~d}$ ). The shPP2A cells grew more slowly than shCont cells. The number of shPP2A cells at day 3 was reduced to $\sim 60 \%$ compared with the number of shCont cells. To further investigate the change in cell morphology, we stained F-actin and nuclei with rhodamine-phalloidin and Hoechst 33342, respectively. The staining clearly showed that shPP2A cells had fewer filopodia and lamellipodia, which are supported by actin fibers (Figure $4 \mathrm{a}$, upper). The phosphorylation levels of FAK were also significantly lower in shPP2A cells, as determined by immunostaining and western blot analysis (Figure $4 \mathrm{a}$, lower and right). Strong intensity, corresponding to phosphorylated FAK, was observed in the projection area in shCont cells, but not shPP2A cells. These observations were in accordance with the result of western blot analysis for phosphorylated FAK. The cells were transfected with the luciferase reporter construct containing the NF- $\kappa \mathrm{B}$ binding site and luciferase activity was measured in collected cell lysates. NF- $\kappa \mathrm{B}$ transcriptional activity was lower in shPP2A cells compared with that in shCont cells (Figure $4 \mathrm{~b}$ ).

\section{Reduction of PP2A Ca Suppresses Cell Migration Ability}

In the transwell migration assay, the number of shPP2A cells that penetrated through the membrane was substantially lower than the number of shCont cells (Figure 4c). In the scratch assay, we evaluated wound closure by measuring the distance between the edges of migrated cells. shPP2A cells also showed decreased ability to migrate into the scratch area (Figure $4 \mathrm{~d}$ ).

\section{Reduction of PP2A Ca Increases Sensitivity to Serum Starvation-Induced Apoptosis}

Next, we examined whether PP2A C $\alpha$ affects mitochondriarelated cell apoptosis. We determined mitochondrial respiratory ability by monitoring OCR in shCont and shPP2A cells. 

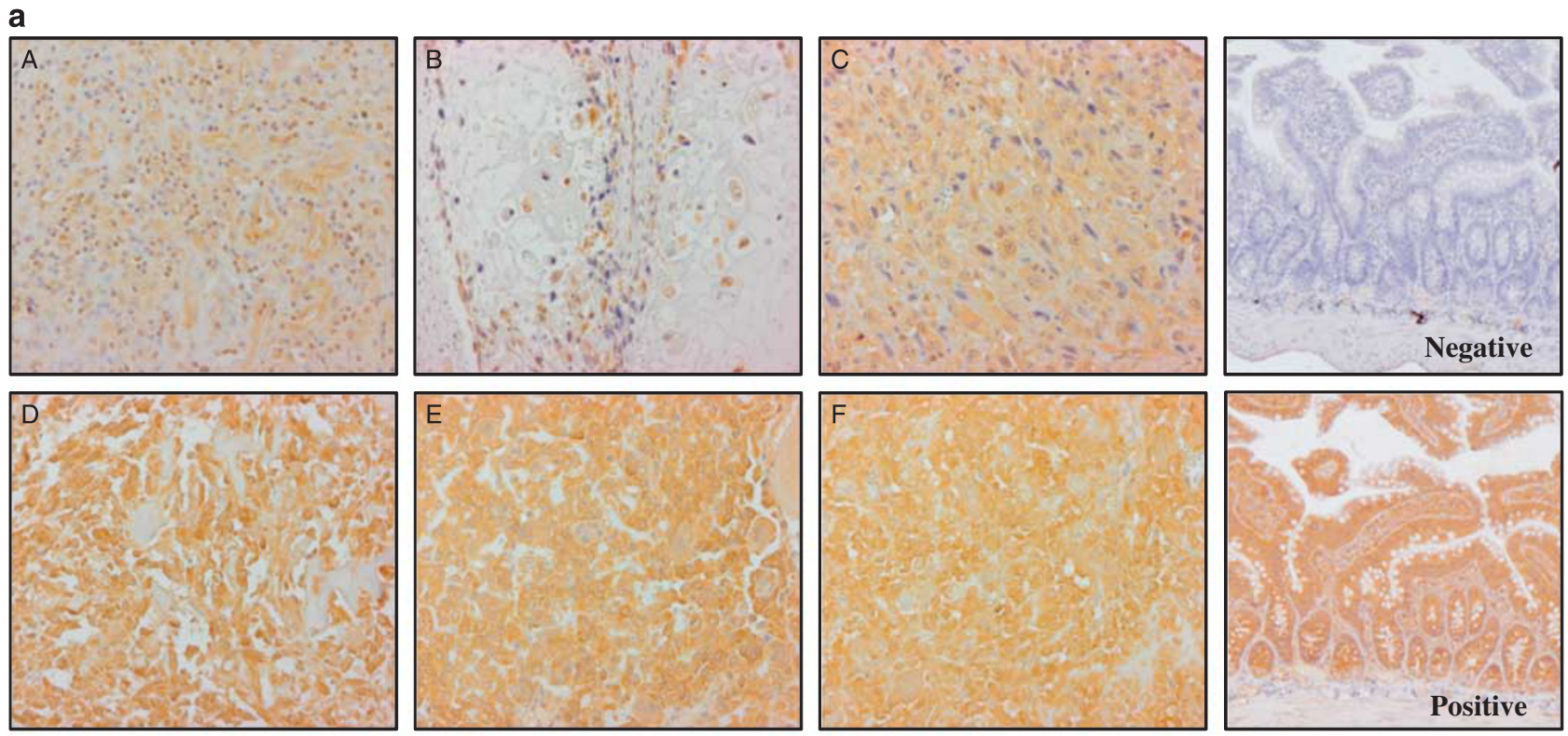

b
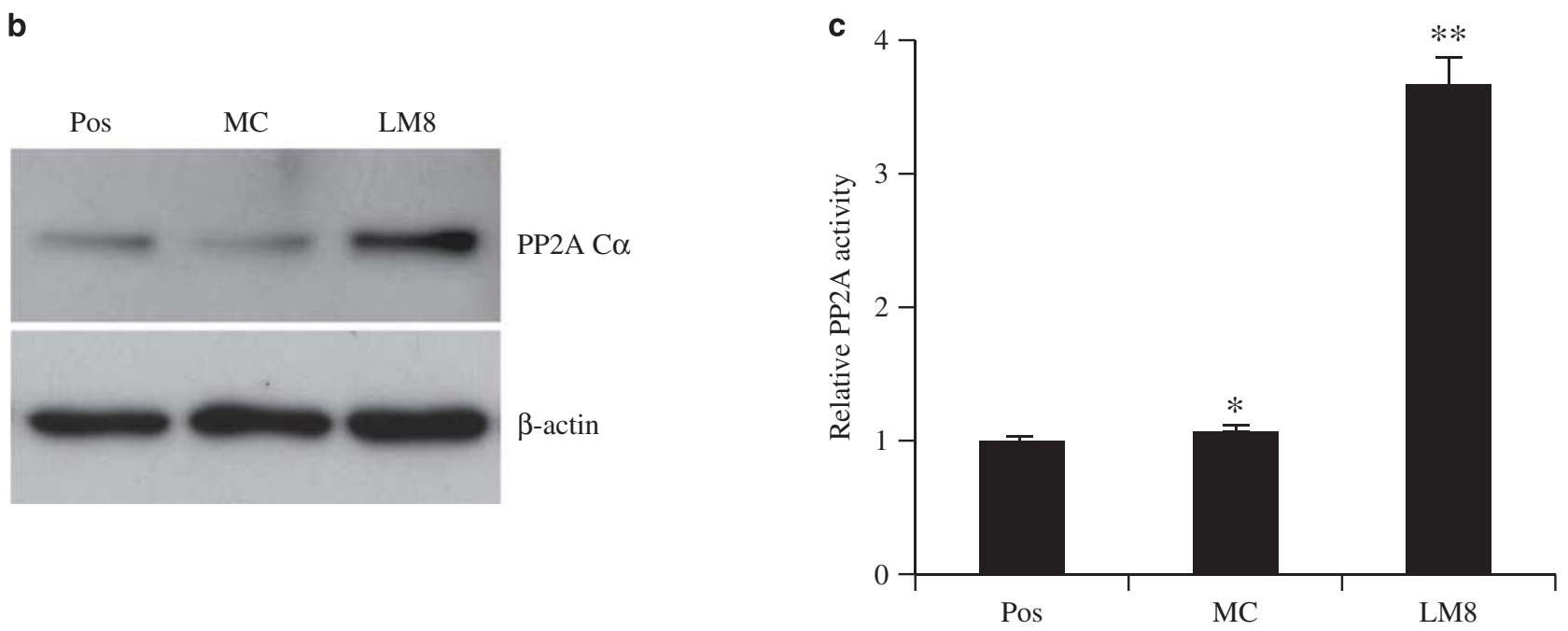

Pos

LM8

Figure 1 PP2A Ca expression in osteosarcoma tissues. (a) PP2A Ca expression was determined by immunohistochemistry using a specific antibody in osteosarcoma tissue array samples. Relative expression levels for PP2A Ca were evaluated by comparing with those in non-malignant granulation tissues (A) on the same slide. Representative images were shown as lower (B), similar (C), and higher (D-F) expression levels of PP2A Ca. Photomicrographs were obtained at $\times 200$ magnification. Representative control images obtained at $\times 100$ magnification were also shown as positive and negative. (b) Total protein was extracted from the cells and protein expression of PP2A Ca and $\beta$-actin were analyzed by western blot. (c) Cell lysates were collected from the cells and PP2A activity was measured in triplicate. Data are expressed relative to the activity of primary osteoblasts as mean \pm s.d. of representative analysis from three separate experiments $\left({ }^{*} P<0.05 ;{ }^{* *} P<0.01\right)$. PP2A, protein phosphatase $2 \mathrm{~A}$.

As shown in Figure 5a, shPP2A cells exhibited lower OCR rates in the untreated condition compared with that in shCont cells $(\sim 15 \%$ decrease at $0 \mathrm{~min})$. The addition of the mitochondrial inhibitor oligomycin similarly reduced oxygen consumption in both cell types (30 and $40 \mathrm{~min}$ ). Maximal OCR was determined by the addition of carbonylcyanide-4trifluorometh-oxyphenylhydrazone (FCCP), an oxidative phosphorylation uncoupler. The addition of FCCP increased OCR levels in shCont cells, whereas OCR levels in shPP2A cells were drastically lower $(\sim 35 \%$ reduction in maximal
OCR). These observations suggest that knockdown of PP2A $\mathrm{C} \alpha$ suppressed mitochondrial respiratory ability.

We further examined whether PP2A C $\alpha$ affects serum deprivation-induced cell apoptosis. Cells were cultured in serum-free medium for $16 \mathrm{~h}$ and PS exposure on the surface of apoptotic cells was measured by flow-cytometry using annexin V/PI staining. The extent of apoptotic cell death was recorded in each part (Figure 5b). The number of annexin V-positive cells was higher in shPP2A cells than that in shCont cells (38.7 vs $21.52 \%)$. In accordance with this result, cleaved forms of 
a

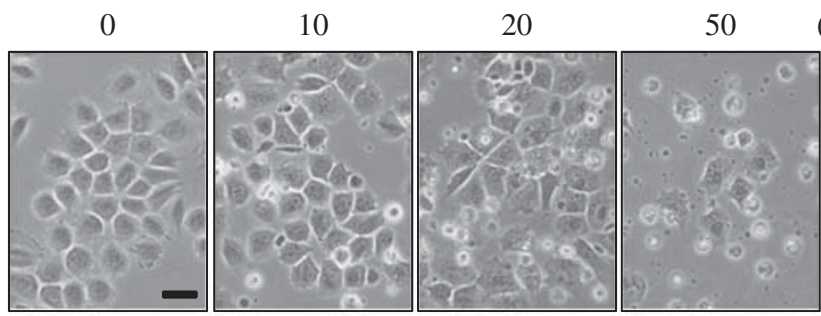

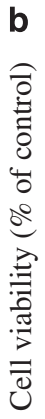

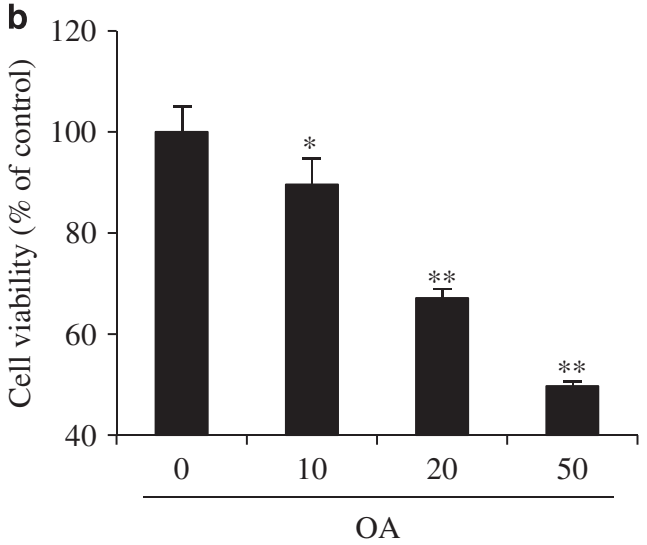

$(\mathrm{nM})$ d

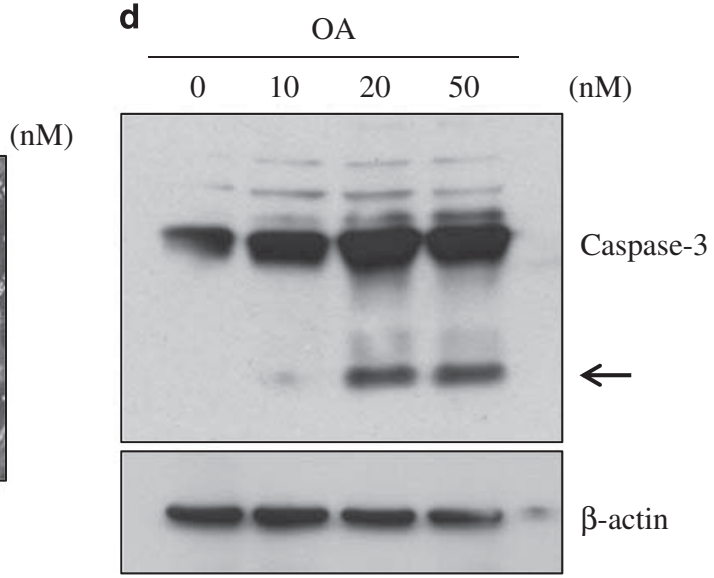

C

$(-)$

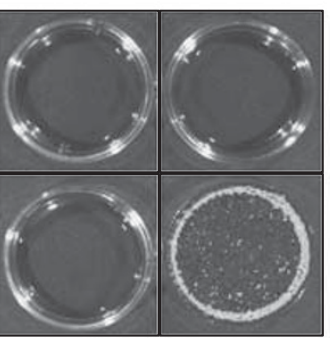

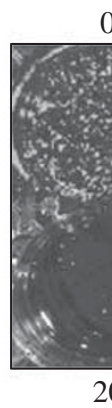

0

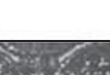

1

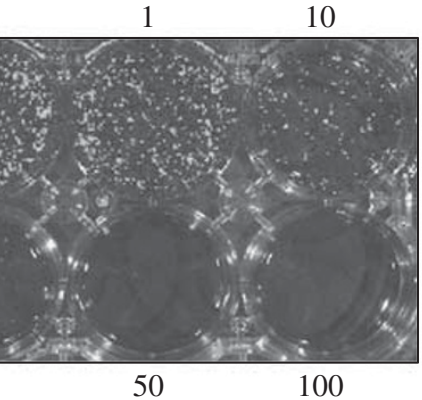

$(\mathrm{nM})$

Figure 2 OA decreases cell viability and induces apoptosis in LM8 cells. LM8 cells were treated with OA at the indicated concentrations. (a) Morphological change was examined under a phase-contrast microscope. Bar represents $10 \mu \mathrm{m}$. (b) Cell viability was quantified using a cell counting kit. Significant differences from the untreated control groups are indicated as ${ }^{*} P<0.01$ and ${ }^{*} P<0.05$, (c, left panel), Luci was added to the culture medium of LM8 cells expressing a luciferase gene (LM8-Luc). Bioluminescence was detected using IVIS. (c, right panel), LM8-Luc cells on a 6-well plate were treated with various concentrations of $\mathrm{OA}$ and bioluminescence intensity was analyzed by addition of luciferin. The experiments were repeated four times and a representative image is shown. (d) Collected cell lysates were subjected to western blot analysis using antibodies against caspase- 3 and $\beta$-actin. The cleaved forms of caspase-3 are pointed by the arrow. OA, okadaic acid; Luci, Luciferin.

caspase-3 and PARP were detected in shPP2A cells at 6 and $24 \mathrm{~h}$, respectively, whereas these cleaved forms were not detected in shCont cells at same time points (Figure 5c). To clarify the mechanism behind the stimulated apoptosis of shPP2A cells by serum deprivation, the status of several mitochondrial proteins was determined by western blot, as shown in Figure 5d. The basal expression levels of antiapoptotic protein BCL-2 was lower in shPP2A cells compared with that in shCont cells. Although BCL-2 was induced by serum deprivation in shPP2A cells, its expression level was still lower at 6 and $12 \mathrm{~h}$ than that in shCont cells. The levels of BAX, $\mathrm{BCL}-\mathrm{XL}$, and $\beta$-actin did not change in either cell type under these conditions. Moreover, the pro-apoptotic protein BIM was similarly induced in both cell types by serum deprivation.

\section{Reduction of PP2A Ca Suppresses Proliferation and Metastasis Abilities of LM8 cells In Vivo}

To examine the role of PP2A C $\alpha$ in the proliferation ability of LM8 cells in vivo, shCont and shPP2A cells were subcutaneously transplanted into the backs of mice. Tumor growth was monitored by the IVIS imaging system. Transplanted shCont and shPP2A cells showed similar levels of bioluminescence intensity at day 0 (Figure 6a, left panel). Although transplanted shCont cells still showed high levels of bioluminescence intensity at day 14 , no signal was obtained from the mice transplanted with shPP2A cells (Figure 6a, right panel).

Because reduction of PP2A C $\alpha$ in LM8 cells suppressed migration ability in vitro, we evaluated the role of PP2A C $\alpha$ in tumor metastasis in vivo. shCont and shPP2A cells injected intraosseously at the proximal part of the tibia shaft were monitored using noninvasive bioluminescence imaging. Figure $6 \mathrm{~b}$ shows representative images of the mice at day 0 and day 14 after injection. Distributed bioluminescence signals were detected in shCont cell-transplanted mice, but not in shPP2A cell-transplanted mice. These results were confirmed by histological analysis of dissected tissues from these mice. As shown in Figure 6c, metastasized tumor cells 
a

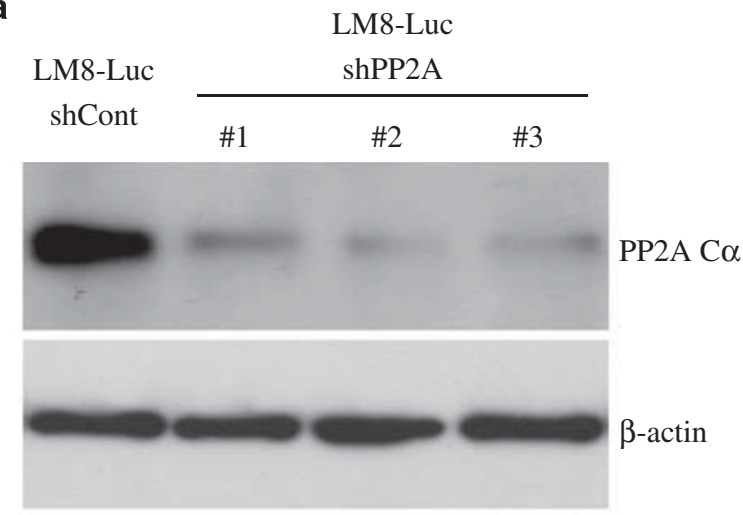

C

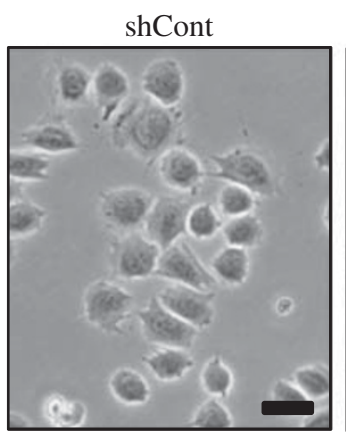

$c$

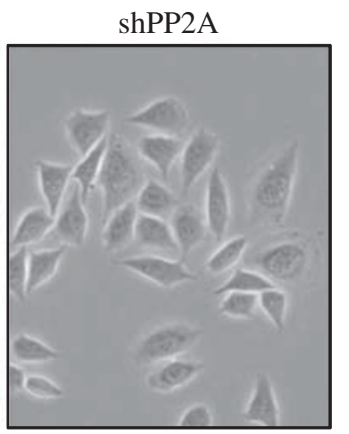

LM8-Luc

b

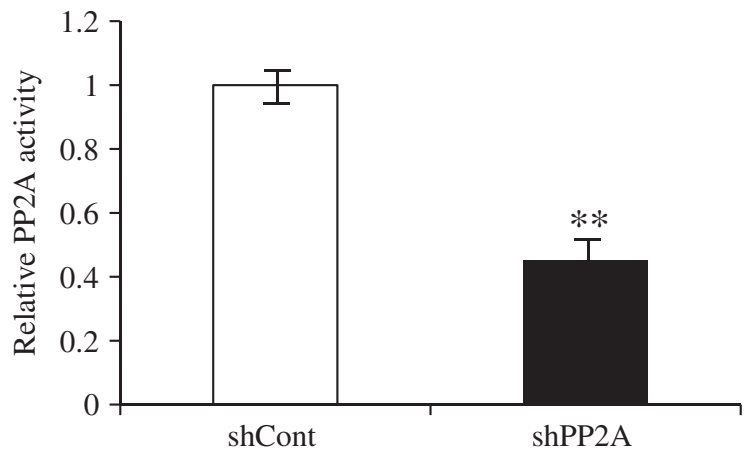

d

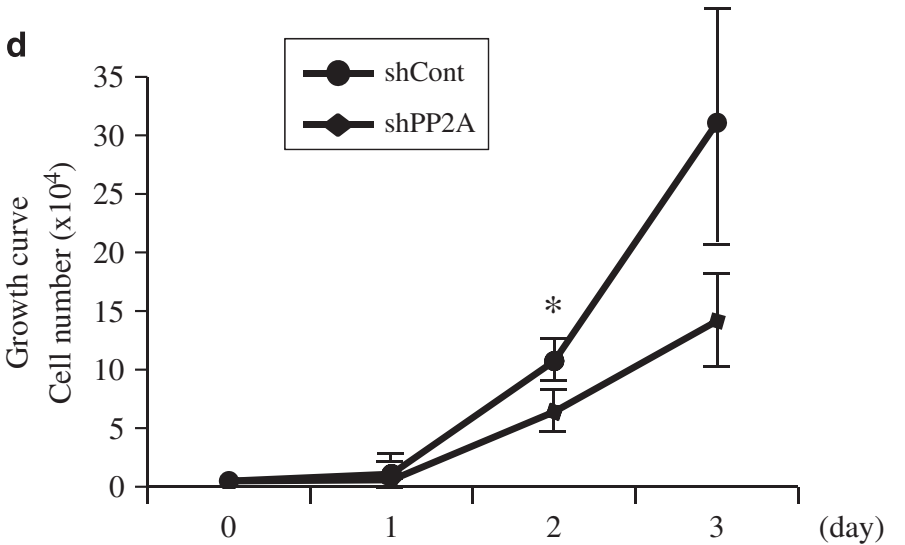

Figure 3 Reduction of PP2A Ca affects cell morphology and proliferation rate of LM8 cells. (a) Establishment of PP2A Ca-knockdown LM8 cells. Luciferase-expressing LM8 cells were transfected with shRNA lentivirus particles producing either a non-target control shRNA or a PP2A Ca shRNA, to construct stable cell lines. Cells were collected and lysed to extract protein, before the lysate was analyzed by western blot with specific antibodies for PP2A C $a$ and $\beta$-actin. (b) Cell lysates were collected from the cells, and PP2A activity was measured in triplicate. Data are expressed relative to the PP2A activity of shCont cells as mean \pm s.d. of representative analysis from three separate experiments $(* * P<0.01)$. (c) Phase-contrast photomicrographs of shCont and shPP2A cells. Bar represents $20 \mu \mathrm{m}$. (d) shCont (closed circles) and shPP2A (closed squares) cells were plated at a density of 5000 per $35-\mathrm{mm}$ dish, cultured for various time periods, and cell numbers were estimated. Each plot shows the average of four different cultures. The experiments were done in triplicate and representative data is presented $\left({ }^{*} P<0.05\right)$. PP2A, protein phosphatase $2 \mathrm{~A}$.

were observed in lung and liver tissues in the samples of shCont cell-transplanted mice, but not in shPP2A celltransplanted mice. Figure $6 \mathrm{~d}$ shows a representative image of the immunostaining of metastasized shCont cells in lung and liver tissues with PCNA antibody. The expression level of PCNA was extremely high in the metastasized cells.

\section{Reduction of PP2A Ca Suppresses Cell Growth and Migration Abilities and Increases the Sensitivity to Serum Starvation-Induced Apoptosis in MG63 Cells}

PP2A C $\alpha$ expression was further examined in human osteosarcoma cell lines, MG63 and SaOS-2. Compared with that in primary osteoblasts and osteoblastic MC3T3-E1 cells, the expression of PP2A C $\alpha$ was significantly higher in MG63 and SaOS- 2 cells as well as LM8 cells (Figure 7a). Knockdown of PP2A C $\alpha$ significantly reduced the cell growth rate in MG63 cells (Figure 7b). In the scratch assay and the transwell migration assay, knockdown of PP2A C $\alpha$ suppressed migration ability in MG63 cells (Figure 7c and d). Furthermore, knockdown of PP2A C $\alpha$ increased the level of cleaved forms of caspase-3 in MG63 cells after serum deprivation for $48 \mathrm{~h}$ (Figure 7e).

\section{DISCUSSION}

Identification of specific tumor biomarkers may help in the investigation and development of novel and effective therapeutic approaches with specific molecular targets to treat patients with osteosarcoma. Here, we explored the role of PP2A C $\alpha$ in osteosarcoma proliferation and metastasis. Our present study demonstrated that PP2A C $\alpha$ expression and PP2A activity are correlated with osteosarcoma proliferation, migration, and metastasis because reduction of PP2A C $\alpha$ attenuates these properties of osteosarcoma. Reduction of PP2A $\mathrm{C} \alpha$ also increased sensitivity to serum deprivationinduced apoptosis in osteosarcoma cells through the downregulation of BCL-2. 
a

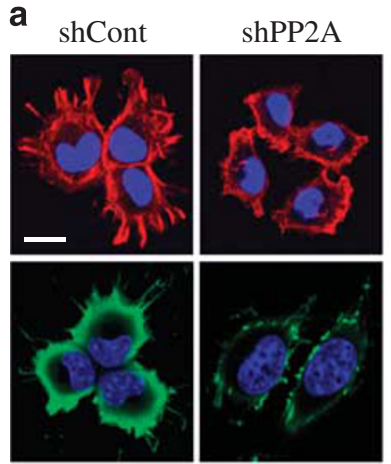

C
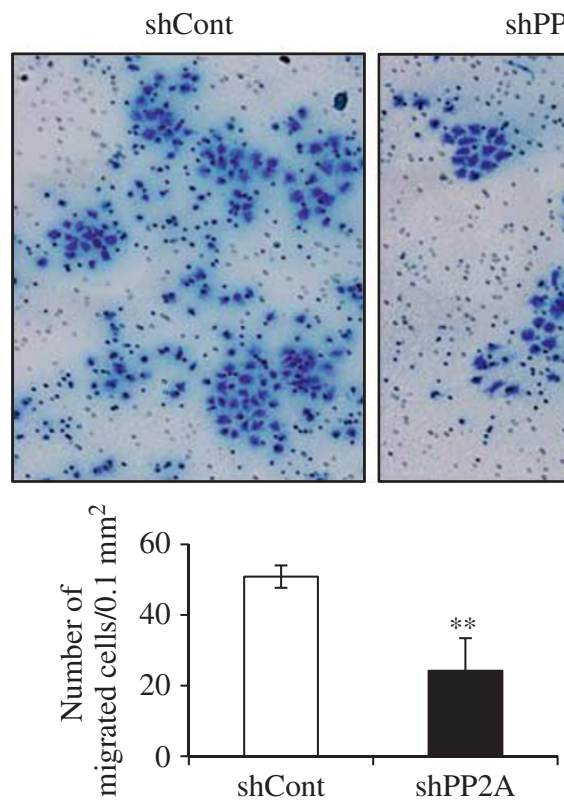

b

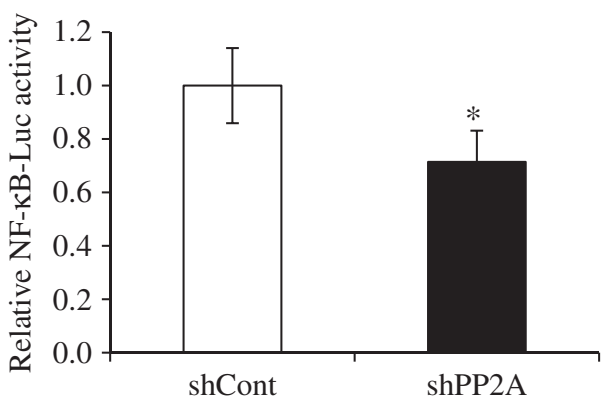

d
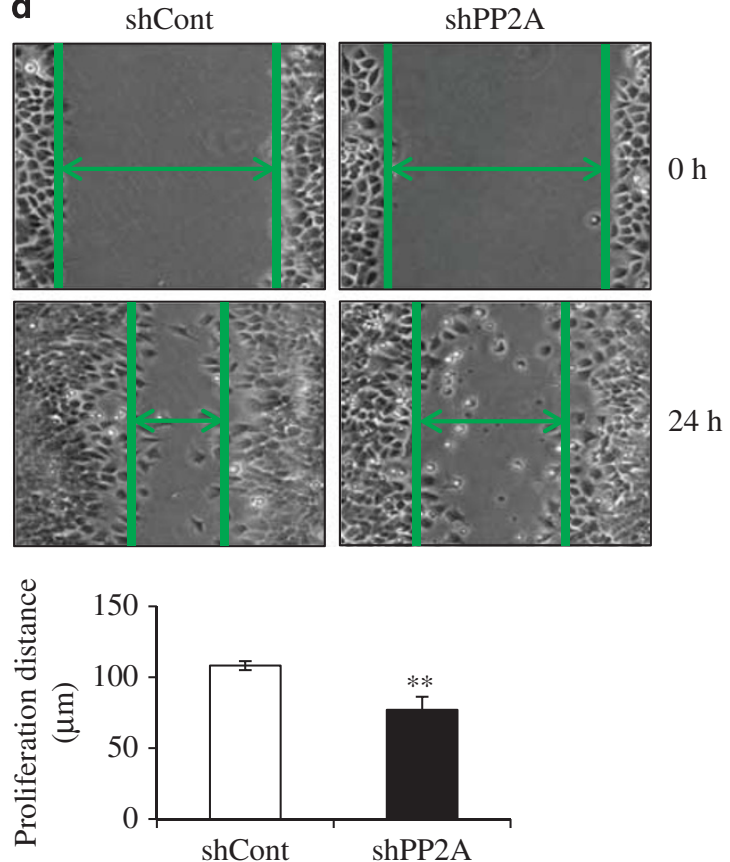

Figure 4 Reduction of PP2A Ca alters F-actin arrangement, phosphorylation of FAK, NF-KB activation, and migration of LM8 cells. (a) F-actin (red), phospho-FA (green), and nuclei (blue) in the cells were visualized using rhodamine-phalloidin, anti-phospho-FAK specific antibody, and $10 \mu \mathrm{g} / \mathrm{ml}$ Hoechst 33342, and then the images were merged. Bar represents $10 \mu \mathrm{m}$ (left). Cell lysates were collected from the cells and the phosphorylation of FAK was determined by western blot. The relative expression of p-FAK/ $\beta$-actin was calculated by computer software (ImageJ) (right). (b) A luciferase construct containing the NF- $K B$ binding site was transfected into the cells. After $24 \mathrm{~h}$, cell lysates were collected and luciferase activity was measured. The experiments were performed in triplicate and representative data was presented $(* * P<0.01)$. (c) Cells were cultured in serum-free medium in the upper chambers of transwell and the lower chambers were filled with medium containing $10 \%$ FBS. After staining with trypan blue, cells that had migrated to the lower surface of the upper chambers were counted under a microscope. Non-migrated cells were scraped with a cotton swab. Representative images are shown. Values are the means \pm s.d.; ${ }^{* *} P<0.01$ : significantly different from the respective control groups. (d) Migration of shPP2A cells was assessed by scratch assay, and compared with that of shCont cells. Representative images of wound closure were taken at 0 and $24 \mathrm{~h}$ after scratch under $\times 100$ magnification. Values are the means \pm s.d.; ${ }^{* *} P<0.01$ : significantly different from the respective control groups. FAK, focal adhesion kinase; PP2A, protein phosphatase $2 \mathrm{~A}$.

First, we examined the expression patterns of PP2A C $\alpha$ in malignant osteosarcoma tissues. In this study, immunohistochemistry showed that a greater number of osteosarcoma tissues express higher amounts of PP2A C $\alpha$ compared with that in benign granulation tissue. However, some malignant osteosarcoma tissues showed moderate or lower levels of PP2A $\mathrm{C} \alpha$ expression. It was reported that there exists a large amount of inter-patient, inter-tumor, and intra- tumor heterogeneity in osteosarcoma. ${ }^{25}$ Therefore, the term 'osteosarcoma' includes a number of different osteosarcoma subtypes, which have diverse histological features and complex genomic organization. ${ }^{25}$ Although the different PP2A C $\alpha$ expression patterns observed in the present study might result from tumor heterogeneity in osteosarcoma, more than half of malignant osteosarcoma tissues showed higher PP2A C $\alpha$ expression. Furthermore, PP2A C $\alpha$ expression and 
a

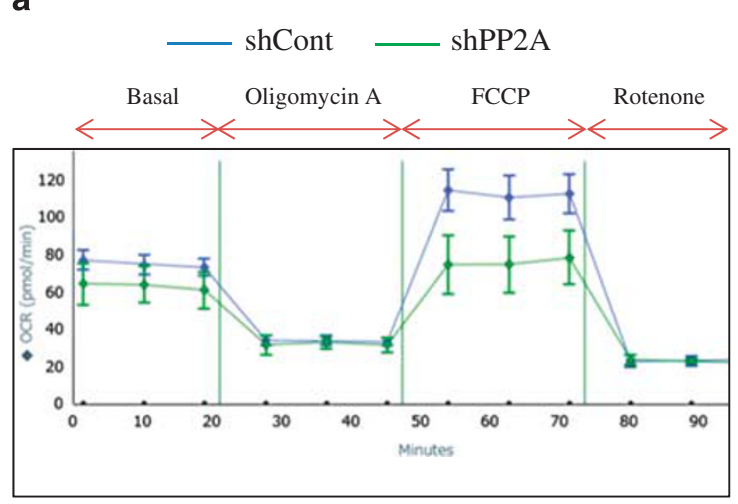

C
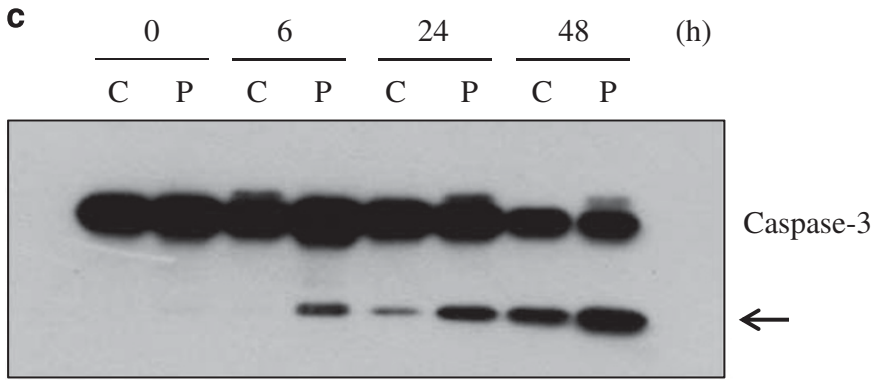

(h)
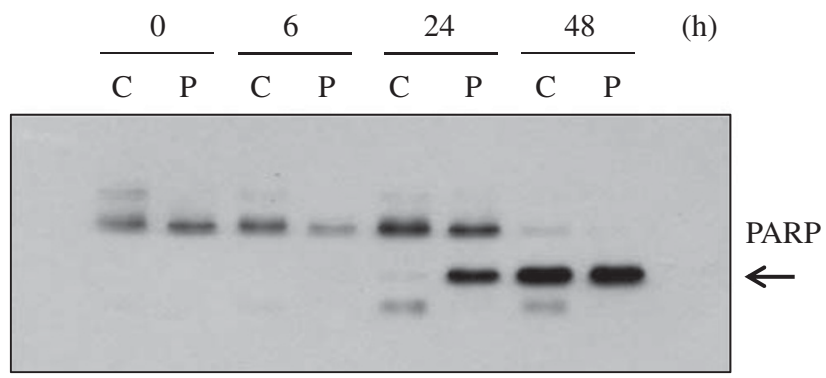

b

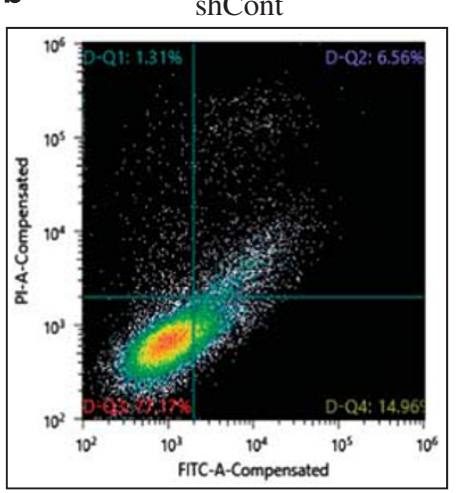

$\operatorname{shPP} 2 \mathrm{~A}$

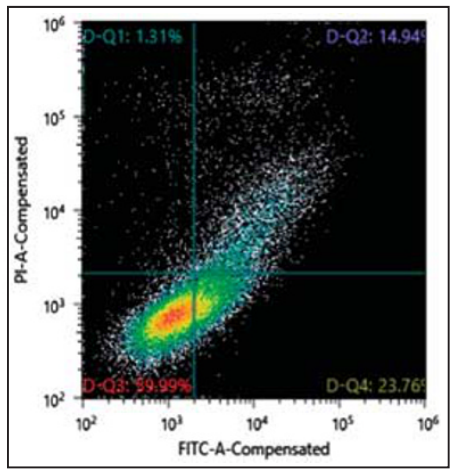

(h)

d
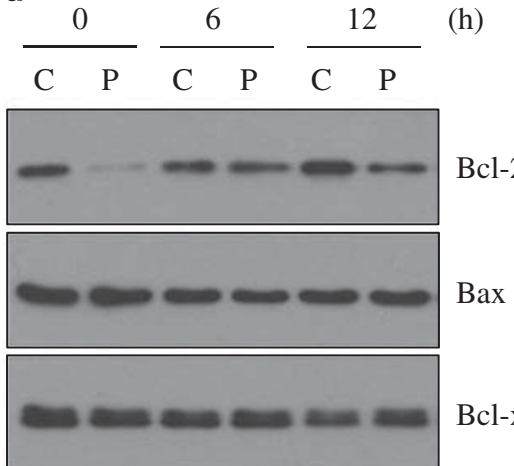

Bcl-xL

Bim

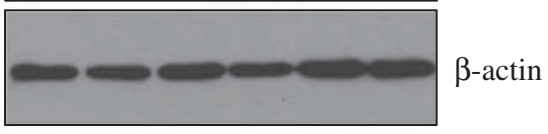

Figure 5 Reduction of PP2A Ca decreases mitochondrial respiration rate and promotes apoptosis. (a) OCR was monitored before (basal level) and after sequential injection of oligomycin, FCCP, and rotenone in shCont and shPP2A cells. (b) Cells were cultured in serum-free medium for $16 \mathrm{~h}$. The percentage of apoptotic cell death was measured by annexin V/PI staining of the cells. The results are representative of three separate experiments yielding similar results. (c,d) Cells were exposed to serum-free medium for the indicated periods and the collected cell lysates were subjected to western blot analysis using specific antibodies. The cleaved forms of caspase-3 and PARP are pointed by the arrow. FCCP, carbonylcyanide-4trifluorometh-oxyphenylhydrazone; OCR, oxygen consumption rate; PP2A, protein phosphatase $2 \mathrm{~A}$.

PP2A activity were examined in primary osteoblasts, immortalized osteoblastic MC3T3-E1 cells, and malignant osteosarcoma LM8 cells. PP2A C $\alpha$ expression and PP2A activity were considerably higher in LM8 cells compared with that in other cells, suggesting that PP2A $\mathrm{C} \alpha$ is involved in the acquisition and progression of malignancy in osteosarcoma cells. Thus, we treated LM8 cells with OA, a potent inhibitor of PP2A, and determined cell morphology and viability. Cell rounding and shrinking were observed in OA-treated LM8 cells in a dose-dependent manner. OA induced cleavage of caspase-3 in LM8 cells, leading to apoptosis.

Next, PP2A C $\alpha$-knockdown cells were established, and cell morphology and proliferation rate were analyzed. Control LM8 cells displayed filopodial and lamellipodial structures neighboring the cell surface, which substantially resembles the phenotype of the original LM8 cells. ${ }^{6}$ In contrast, apparent filopodial and lamellipodial structures disappeared in PP2A $\mathrm{C} \alpha$-knockdown cells. Moreover, PP2A C $\alpha$-knockdown decreased the proliferation rate of LM8 cells. LM8 cells were established as an osteosarcoma cell line with high metastatic potential to the lungs. During this process, the original osteosarcoma cells acquired filopodial and lamellipodial structures on their surface. ${ }^{6}$ It was reported that genistein, an isoflavone phytoestrogen found in soy, inhibited tumor growth, and decreased the number of spindle-shaped cells in LM8 cultures. ${ }^{26}$ These findings strongly suggest that PP2A C $\alpha$ has an important role in the acquisition of distinct cell morphology in LM8 cells. In addition, LM8 cells display higher levels of FAK phosphorylation at focal adhesion sites, ${ }^{8}$ which has key roles in cell migration and metastasis of tumor 
a

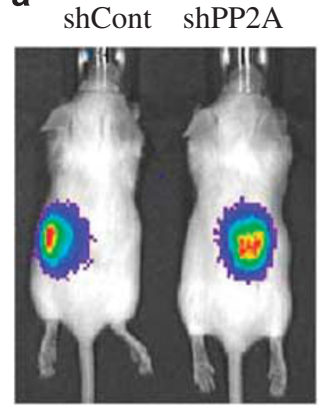

Day 0

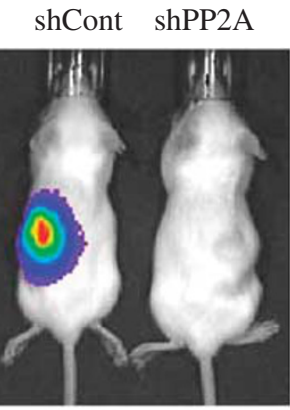

Day 14

b

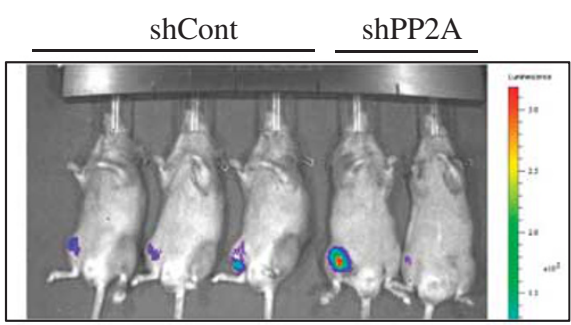

2 weeks

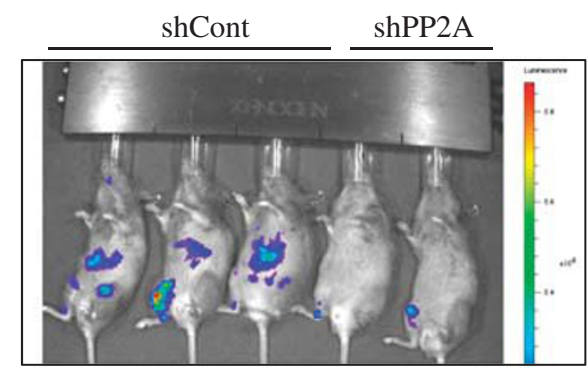

C
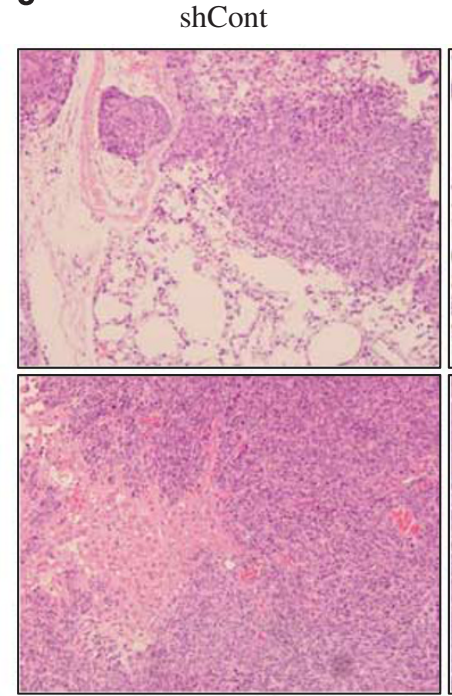

d
shPP2 A

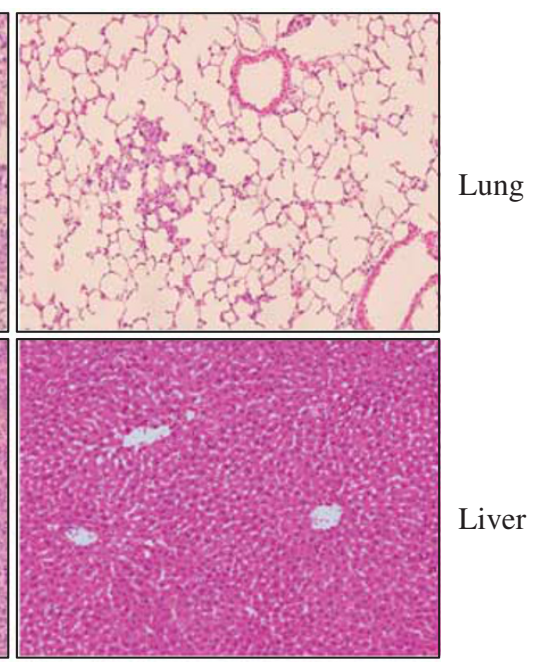

PCNA

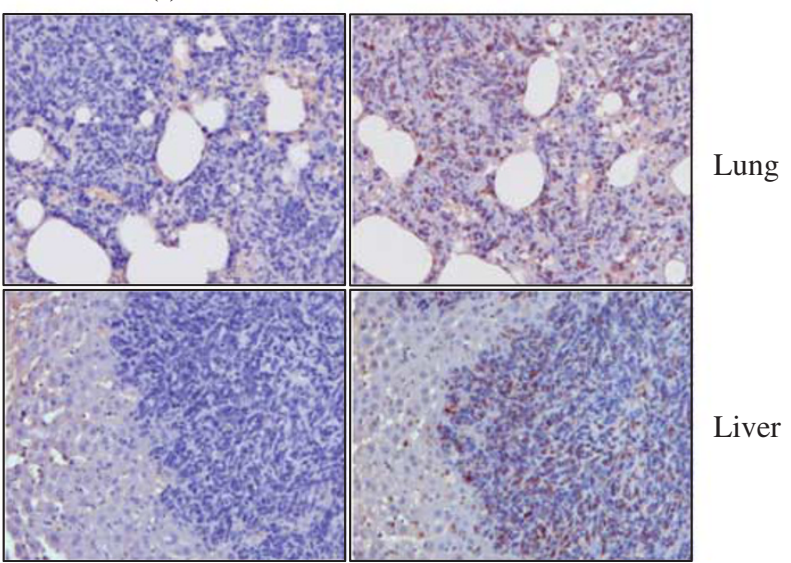

Figure 6 Reduction of PP2A Ca suppresses proliferation and metastasis of LM8 cells in vivo. The cells were subcutaneously transplanted into the back (a) or tibia bone marrow (b-d) of each mouse. Tumor growth was followed using bioluminescence imaging in vivo and ex vivo. (a) Representative images at day 0 and 14 after transplantation are shown. Bioluminescence intensity is indicative of tumor volume. (b) Tumor growth and metastasis were followed using imaging bioluminescence. Representative images at day 0 and 14 are shown. (c) Representative histological images of pulmonary and hepatic metastasis are shown (HE staining). Photomicrographs were obtained at $\times 200$ magnification. In the control group, LM 8 cells formed large nodules and invaded into lung alveoli and liver parenchyma. In the shPP2A group, tissue architectures were preserved and tumor nodules were considerably smaller. (d) Representative image of the immunostaining of metastasized shCont cells in lung and liver tissues with PCNA antibody. PP2A, protein phosphatase $2 \mathrm{~A}$.

cells. NF- $\kappa \mathrm{B}$ has also been reported to have a central and specific role in the regulation of metastasis in LM8 cells, because NF- $\kappa \mathrm{B}$ decoy transfection inhibited pulmonary metastasis of these cells. ${ }^{27}$ In the present study, phosphorylation levels of FAK at the cell protrusion region and NF- $\kappa \mathrm{B}$ activity were suppressed in shPP2A cells, suggesting that PP2A $\mathrm{C} \alpha$ regulates cell migration and metastasis by controlling FAK and NF- $\kappa \mathrm{B}$ activity.

Mitochondria have important roles in cell energy production and metabolism, and mitochondrial dysfunction has been linked to a wide array of human diseases including tumor growth. ${ }^{28,29}$ High oxygen consumption through mitochondrial respiration was observed in shCont cells. Nonetheless, reduction of PP2A C $\alpha$ led to suppressed mitochondrial respiratory capacity at the basal and maximal level. It was demonstrated that low metastatic tumor cells have lower oxygen consumption than high metastatic tumors, including osteosarcoma. ${ }^{30,31}$ Although many studies reported a shift from oxidative phosphorylation to glycolysis to provide energy for cellular activities in cancer cells, ${ }^{32}$ cancer cells have also been reported to rely on oxidative phosphorylation and mitochondrial bioenergetics pathways for anabolism and energy production. ${ }^{33}$ Our present finding indicates that PP2A $\mathrm{C} \alpha$ is involved in the regulation of mitochondrial respiratory 
a

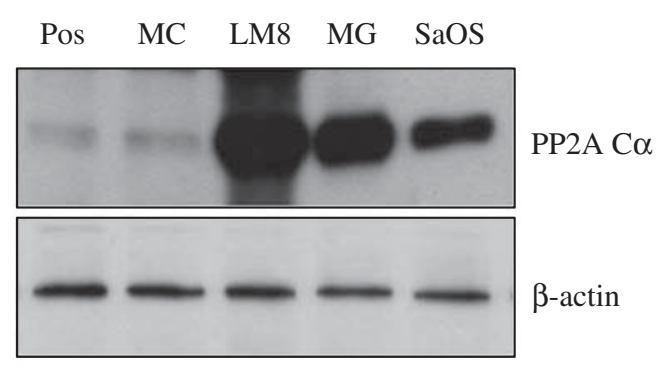

c

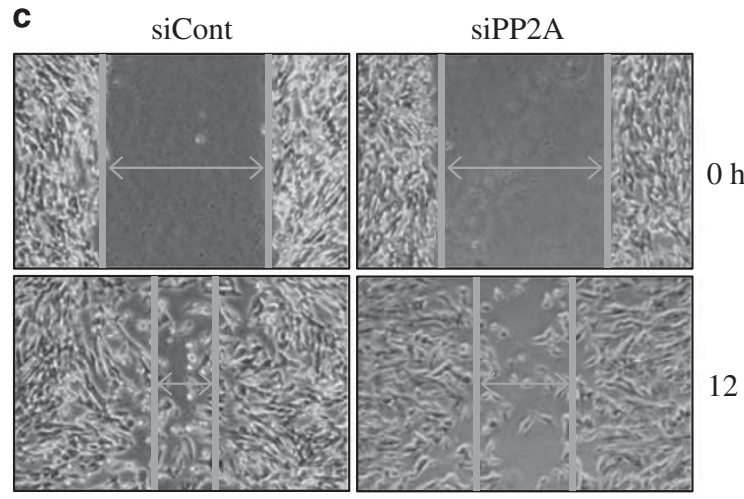

b
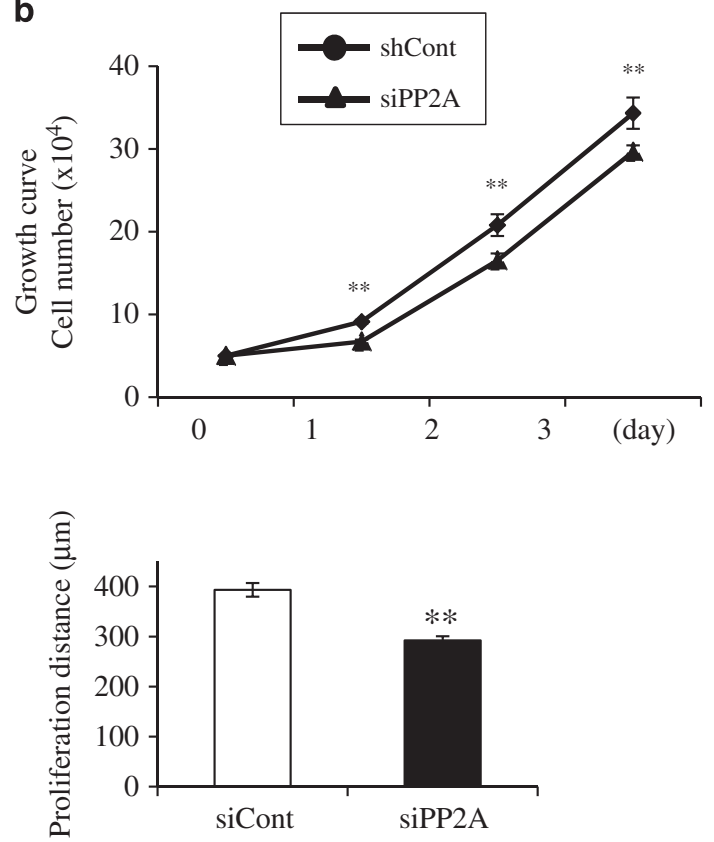

e

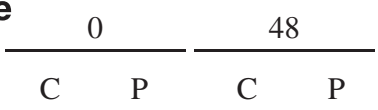

(h)

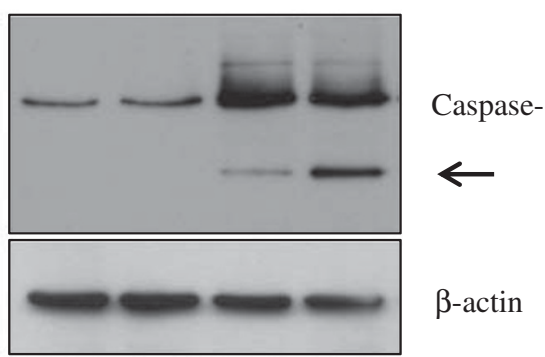

Figure 7 Reduction of PP2A Ca suppresses cell growth rate and migration ability of MG63 cells. (a) Total protein was extracted from the cells and protein expression of PP2A Ca and $\beta$-actin were analyzed by western blot. (b) siCont (closed circles) and siPP2A (closed triangles) cells were plated at a density of 50000 per 35-mm dish, cultured for various time periods, and cell numbers were estimated. Each plot shows the average of four different cultures. The experiments were done in triplicate and representative data is presented $\left({ }^{* *} P<0.01\right)$. (c) Migration of siPP2A cells was assessed by scratch assay, and compared with that of siCont cells. Representative images of wound closure were taken at 0 and $12 \mathrm{~h}$ after scratch under $\times 100$ magnification. Values are the means \pm s.d.; ${ }^{* *} P<0.01$ : significantly different from the respective control groups. (d) Cells were cultured in serum-free medium in the upper chambers of transwell; the lower chambers were filled with medium containing $10 \%$ FBS. After staining with trypan blue, cells that had migrated to the lower surface of the upper chambers were counted under a microscope. Non-migrated cells were scraped with a cotton swab. Representative images are shown. Values are the means \pm s.d.; ${ }^{* *} P<0.01$, significantly different from the respective control groups. (e) Cells were exposed to serum-free medium for the indicated periods and the collected cell lysates were subjected to western blot using specific antibodies. The cleaved forms of caspase-3 are pointed by the arrow. PP2A, protein phosphatase $2 \mathrm{~A}$.

capacity. The molecular mechanism by which reduction of PP2A C $\alpha$ attenuates mitochondrial aerobic metabolism remains to be determined.

We next investigated sensitivity to serum deprivationinduced apoptosis in shCont and shPP2A cells. The number of annexin V-positive cells was higher in shPP2A cells after serum deprivation compared with that in shCont cells. Cleaved caspase-3 and PARP were also detected at an earlier time point in shPP2A cells. These observations indicate that
shPP2A cells are more sensitive to serum deprivation-induced apoptosis. Serum deprivation-induced apoptosis is mainly regulated by mitochondrial proteins including those of the BCL-2 family. In the present study, BCL-2 expression was lower in shPP2A cells than in shCont cells at the basal level. Among members of this family, BCL-2 and BAX are central regulators of apoptosis that inhibit (BCL-2) or stimulate (BAX) cell death. ${ }^{34}$ BCL-2 and BAX are able to control apoptosis competitively or independently, ${ }^{35,36}$ therefore, the ratio 
of BCL-2/BAX is an important indicator of apoptosis. ${ }^{37}$ Owing to its anti-apoptotic properties, BCL-2 is overexpressed in a variety of cancers, thereby allowing them to escape from various apoptotic signals. ${ }^{38}$ It was also reported that overexpression of BCL-2 increases mitochondrial respiration. ${ }^{38,39}$ These findings suggest that reduction of BCL-2 expression in shPP2A cells increases the sensitivity to serum deprivation-induced apoptosis.

Finally, we examined the role of PP2A C $\alpha$ in LM8 cell migration using traditional in vitro approaches. Reduction of PP2A $\mathrm{C} \alpha$ decreased the migration ability of LM8 cells. Mice transplanted with shCont cells in the tibia bone marrow region exhibited metastasis to lung and liver at high frequency. However, metastasis to distant organs was barely detectable in the mice transplanted with shPP2A cells. These observations further indicate that PP2A C $\alpha$ has critical roles in the abilities of migration and metastasis in osteosarcoma cells.

A number of studies have highlighted the role of PP2A as a tumor suppressor. PP2A has complex roles in cells, depending upon the state of specific subunits, cell type, and biological context. Given the prominent activation of kinase in various cancer cells, ${ }^{40} \mathrm{PP} 2 \mathrm{~A}$ is considered a tumor suppressor by virtue of its dephosphorylation activity. ${ }^{11,41}$ In fact, the inhibition of PP2A by OA promotes tumor growth in mice. ${ }^{42-44}$ Similarly, it was established that the viral SV40 small tumor antigen, which is required for cellular transformation in human cells, could alter PP2A activity. ${ }^{45,46}$ Finally, mutations have been identified in different components of the PP2A holoenzyme complex, which have been linked to a variety of primary human tumors. ${ }^{11,47}$ In contrast, some reports demonstrated the role of $\mathrm{PP} 2 \mathrm{~A}$ as a tumor promoter; thus, inhibition of $\mathrm{PP} 2 \mathrm{~A}$ increases sensitivity to anticancer drugs and/or induces cell death. PP2A inhibitors including LB-100 and calyculin A have been evaluated as sensitizers for chemotherapy or radiotherapy in several malignancies. ${ }^{48-50}$ Cantharidin, another PP2A inhibitor, suppresses the migration and growth of pancreatic and breast cancer cells. ${ }^{48,51-53}$ We also previously reported that OA induced apoptosis in several types of cell line including osteosarcoma cells through NF- $\kappa \mathrm{B}$ and PKR pathways. ${ }^{17,18}$ Furthermore, genistein attenuates the proliferative rate and invasive potential of LM8 cells. ${ }^{54}$ In this process, treatment of LM8 cells with genistein induces morphological changes to a fibroblast-like shape, which is considerably similar to the effect of reduction of PP2A C $\alpha$ expression in these cells. These observations suggest that $\mathrm{PP} 2 \mathrm{~A}$ has various roles in different types of tumor cells.

In summary, our results show that PP2A C $\alpha$ is an important factor for proliferation, migration, and metastasis of osteosarcoma cells. Increases in PP2A activity and PP2A C $\alpha$ expression were observed in malignant osteosarcoma LM8 cells as well as human osteosarcoma MG63 and SaOS cells. Reduction of PP2A C $\alpha$ induced morphological change in these cells and decreased the activation of NF- $\kappa \mathrm{B}$ and FAK, followed by the suppression of proliferation and migration in vitro. In addition, reduction of $\mathrm{PP} 2 \mathrm{~A} \mathrm{C} \alpha$ in LM8 cells attenuates their proliferation and metastasis ability in vivo. Repressive effects of PP2A C $\alpha$ knockdown on proliferation and metastasis ability were also observed in human osteosarcoma MG63 cells. PP2A is one of the most critical phosphatases in cancer cells, thus an alteration of its activity may not only influence the phosphorylation and dephosphorylation balance of certain proteins, but potentially also many components of the serine/threonine kinase signaling network. This diversity of PP2A function in tumors suggests that, in certain circumstances, targeting PP2A C $\alpha$ expression might be an effective anti-cancer strategy.

\section{ACKNOWLEDGMENTS}

We would like to thank Dr Omar MM Rodis for help in the preparation of this manuscript. We also thank Drs Hideaki Horikawa and Shigeo Misawa in the Support Center for Advanced Medical Sciences, Institute of Health Biosciences, Tokushima University Graduate School, for technical support. This study was supported by grants from the Grant-in-Aid for Scientific Research from the Ministry of Education, Science, Sports, and Culture of Japan (23592703, HO), the Ichiro Kanehara Foundation for the Promotion of Medical Sciences and Medical Care, Takeda Science Foundation, and the Nakatomi Foundation.

\section{DISCLOSURE/CONFLICT OF INTEREST}

The authors declare no conflict of interest.

1. Dome JS, Schwartz CL. Osteosarcoma. Cancer Treat Res 1997;92: 215-251.

2. Bacci G, Longhi A, Versari M, et al. Prognostic factors for osteosarcoma of the extremity treated with neoadjuvant chemotherapy: 15-year experience in 789 patients treated at a single institution. Cancer 2006;106:1154-1161.

3. Ferguson WS, Goorin AM. Current treatment of osteosarcoma. Cancer Invest 2001;19:292-315.

4. Saeter G, Wiebe T, Wiklund T, et al. Chemotherapy in osteosarcoma. The Scandinavian Sarcoma Group experience. Acta Orthop Scand Suppl 1999;285:74-82.

5. Mori S, Ueda T, Kuratsu S, et al. Suppression of pulmonary metastasis by angiogenesis inhibitor TNP-470 in murine osteosarcoma. Int J Cancer 1995;61:148-152.

6. Asai T, Ueda $\mathrm{T}$, Itoh $\mathrm{K}$, et al. Establishment and characterization of a murine osteosarcoma cell line (LM8) with high metastatic potential to the lung. Int J Cancer 1998;76:418-422.

7. Tomonaga M, Hashimoto N, Tokunaga $F$, et al. Activation of nuclear factor- $\kappa$ B by linear ubiquitin chain assembly complex contributes to lung metastasis of osteosarcoma cells. Int J Oncol 2012;40:409-417.

8. Yui $\mathrm{Y}$, Itoh $\mathrm{K}$, Yoshioka $\mathrm{K}$, et al. Mesenchymal mode of migration participates in pulmonary metastasis of mouse osteosarcoma LM8. Clin Exp Metastasis 2010;27:619-630.

9. Shi Y. Assembly and structure of protein phosphatase $2 A$. Sci China C Life Sci 2009;52:135-146.

10. Shi Y. Serine/threonine phosphatases: mechanism through structure. Cell 2009;139:468-484.

11. Westermarck J, Hahn WC. Multiple pathways regulated by the tumor suppressor PP2A in transformation. Trends Mol Med 2008;14:152-160.

12. Janssens V, Goris J. Protein phosphatase $2 A$ : a highly regulated family of serine/threonine phosphatases implicated in cell growth and signalling. Biochem J 2001;353:417-439.

13. Janssens V, Longin S, Goris J. PP2A holoenzyme assembly: in cauda venenum (the sting is in the tail). Trends Biochem Sci 2008;33:113-121.

14. Gotz J, Probst A, Ehler E, et al. Delayed embryonic lethality in mice lacking protein phosphatase $2 \mathrm{~A}$ catalytic subunit $\mathrm{Ca}$. Proc Natl Acad Sci U S A 1998;95:12370-12375. 
15. Gotz J, Probst A, Mistl C, et al. Distinct role of protein phosphatase $2 A$ subunit $\mathrm{C} a$ in the regulation of $\mathrm{E}$-cadherin and $\beta$-catenin during development. Mech Dev 2000;93:83-93.

16. Morimoto $\mathrm{H}$, Ozaki $\mathrm{A}$, Okamura $\mathrm{H}$, et al. Okadaic acid induces tyrosine phosphorylation of $\mathrm{I}_{\kappa} \mathrm{Ba}$ that mediated by PKR pathway in human osteoblastic MG63 cells. Mol Cell Biochem 2005;276:211-217.

17. Haneji T, Hirashima K, Teramachi J, et al. Okadaic acid activates the PKR pathway and induces apoptosis through PKR stimulation in MG63 osteoblast-like cells. Int J Oncol 2013;42:1904-1910.

18. Ozaki A, Morimoto $\mathrm{H}$, Tanaka $\mathrm{H}$, et al. Okadaic acid induces phosphorylation of p65NF- $K B$ on serine 536 and activates NF- $\kappa$ B transcriptional activity in human osteoblastic MG63 cells. J Cell Biochem 2006;99:1275-1284

19. Okamura H, Yoshida K, Ochiai K, et al. Reduction of protein phosphatase 2A Ca enhances bone formation and osteoblast differentiation through the expression of bone-specific transcription factor Osterix. Bone 2011;49:368-375.

20. Okamura H, Yoshida K, Yang D, et al. Protein phosphatase 2A Ca regulates osteoblast differentiation and the expressions of bone sialoprotein and osteocalcin via osterix transcription factor. J Cell Physiol 2013;228:1031-1037.

21. Okamura H, Yang D, Yoshida $\mathrm{K}$, et al. Protein phosphatase $2 \mathrm{~A} \mathrm{Ca}$ is involved in osteoclastogenesis by regulating RANKL and OPG expression in osteoblasts. FEBS Lett 2013;587:48-53.

22. Kvansakul M, Hinds MG. The Bcl-2 family: structures, interactions and targets for drug discovery. Apoptosis 2015;20:136-150.

23. Xiong $\mathrm{S}, \mathrm{Mu} \mathrm{T}$, Wang $\mathrm{G}$, et al. Mitochondria-mediated apoptosis in mammals. Protein Cell 2014;5:737-749.

24. Wen X, Lin ZQ, Liu B, et al. Caspase-mediated programmed cell death pathways as potential therapeutic targets in cancer. Cell Prolif 2012;45: 217-224.

25. Botter SM, Neri D, Fuchs B. Recent advances in osteosarcoma. Curr Opin Pharmacol 2014;16:15-23.

26. Nakamura A, Aizawa J, Sakayama K, et al. Genistein inhibits cell invasion and motility by inducing cell differentiation in murine osteosarcoma cell line LM8. BMC Cell Biol 2012;13:24.

27. Nishimura A, Akeda K, Matsubara T, et al. Transfection of NF- $\kappa$ B decoy oligodeoxynucleotide suppresses pulmonary metastasis by murine osteosarcoma. Cancer Gene Ther 2011;18:250-259.

28. Schapira AH. Mitochondrial diseases. Lancet 2012;379:1825-1834.

29. Raimundo N, Baysal BE, Shadel GS. Revisiting the TCA cycle: signaling to tumor formation. Trends Mol Med 2011;17:641-649.

30. Ren L, Hong SH, Chen QR, et al. Dysregulation of ezrin phosphorylation prevents metastasis and alters cellular metabolism in osteosarcoma. Cancer Res 2012;72:1001-1012.

31. Zheng H, Li S, Hsu P, et al. Induction of a tumor-associated activating mutation in protein tyrosine phosphatase Ptpn11 (Shp2) enhances mitochondrial metabolism, leading to oxidative stress and senescence. J Biol Chem 2013;288:25727-25738.

32. Kim JW, Dang CV. Cancer's molecular sweet tooth and the Warburg effect. Cancer Res 2006;66:8927-8930.

33. Jose C, Bellance N, Rossignol R. Choosing between glycolysis and oxidative phosphorylation: a tumor's dilemma? Biochim Biophys Acta 2011;1807:552-561.

34. Wiren KM, Toombs AR, Semirale AA, et al. Osteoblast and Osteocyte Apoptosis Associated with Androgen Action in Bone: Requirement of Increased Bax/Bcl-2 Ratio. Bone: United States, 2006, pp 637-651.
35. Hotchkiss RS, Swanson PE, Knudson CM, et al. Overexpression of $\mathrm{BCl}-2$ in transgenic mice decreases apoptosis and improves survival in sepsis. J Immunol 1999;162:4148-4156.

36. Knudson CM, Korsmeyer SJ. Bcl-2 and Bax function independently to regulate cell death. Nat Genet 1997;16:358-363.

37. Zeren $\mathrm{T}$, Inan S, Vatansever HS, et al. Significance of apoptosis related proteins on malignant transformation of ovarian tumors: A comparison between $\mathrm{Bcl}-2 / \mathrm{Bax}$ ratio and p53 immunoreactivity. Acta Histochem 2014;116:1251-1258.

38. Krishna S, Low IC, Pervaiz S. Regulation of mitochondrial metabolism: yet another facet in the biology of the oncoprotein $\mathrm{Bcl}-2$. Biochem J 2011;435:545-551.

39. Chen ZX, Pervaiz S. BCL-2: pro-or anti-oxidant? Front Biosci (Elite Ed) 2009;1:263-268.

40. Seshacharyulu P, Pandey P, Datta K, et al. Phosphatase: PP2A structural importance, regulation and its aberrant expression in cancer. Cancer Lett 2013;335:9-18.

41. Mumby M. PP2A: unveiling a reluctant tumor suppressor. Cell 2007; 130:21-24.

42. Suganuma $M$, Fujiki $H$, Suguri $H$, et al. Okadaic acid: an additional non-phorbol-12-tetradecanoate-13-acetate-type tumor promoter. Proc Natl Acad Sci U S A 1988;85:1768-1771.

43. Suganuma M, Fujiki $H$, Furuya-Suguri $H$, et al. Calyculin $A$, an inhibitor of protein phosphatases, a potent tumor promoter on CD-1 mouse skin. Cancer Res 1990;50:3521-3525.

44. Schonthal AH. Role of PP2A in intracellular signal transduction pathways. Front Biosci 1998;3:D1262-D1273.

45. Sablina AA, Hahn WC. SV40 small T antigen and PP2A phosphatase in cell transformation. Cancer Metastasis Rev 2008;27:137-146.

46. Arroyo JD, Hahn WC. Involvement of PP2A in viral and cellular transformation. Oncogene 2005;24:7746-7755.

47. Eichhorn PJ, Creyghton MP, Bernards R. Protein phosphatase 2A regulatory subunits and cancer. Biochim Biophys Acta 2009;1795:1-15.

48. Bai X, Zhi X, Zhang Q, et al. Inhibition of protein phosphatase 2A sensitizes pancreatic cancer to chemotherapy by increasing drug perfusion via HIF-1 $a$-VEGF mediated angiogenesis. Cancer Lett 2014;355: 281-287.

49. Bai $X L$, Zhang $Q, Y e ~ L Y$, et al. Inhibition of protein phosphatase $2 A$ enhances cytotoxicity and accessibility of chemotherapeutic drugs to hepatocellular carcinomas. Mol Cancer Ther 2014;13:2062-2072.

50. Dedinszki D, Kiss A, Markasz L, et al. Inhibition of protein phosphatase1 and $-2 A$ decreases the chemosensitivity of leukemic cells to chemotherapeutic drugs. Cell Signal 2015;27:363-372.

51. Wu MY, Xie X, Xu ZK, et al. PP2A inhibitors suppress migration and growth of PANC-1 pancreatic cancer cells through inhibition on the Wnt/ $\beta$-catenin pathway by phosphorylation and degradation of $\beta$-catenin. Oncol Rep 2014;32:513-522.

52. Li W, Chen Z, Gong FR, et al. Growth of the pancreatic cancer cell line PANC-1 is inhibited by protein phosphatase $2 \mathrm{~A}$ inhibitors through overactivation of the c-Jun $\mathrm{N}$-terminal kinase pathway. Eur J Cancer 2011;47:2654-2664.

53. Shou LM, Zhang QY, Li W, et al. Cantharidin and norcantharidin inhibit the ability of MCF-7 cells to adhere to platelets via protein kinase C pathway-dependent downregulation of $a 2$ integrin. Oncol Rep 2013;30:1059-1066.

54. Ishimi Y, Miyaura C, Jin CH, et al. IL- 6 is produced by osteoblasts and induces bone resorption. J Immunol 1990;145:3297-3303. 